\title{
A Lipid Mediator Hepoxilin A3 Is a Natural Inducer of Neutrophil Extracellular Traps in Human Neutrophils
}

\author{
David N. Douda, ${ }^{1,2,3}$ Hartmut Grasemann,, ${ }^{1,4,5}$ Cecil Pace-Asciak, ${ }^{1,6}$ and Nades Palaniyar ${ }^{1,2,5}$ \\ ${ }^{1}$ Lung Innate Immunity Research Laboratory, Program in Physiology \& Experimental Medicine, \\ The Hospital for Sick Children Research Institute, Toronto, ON, Canada M5G 0A4 \\ ${ }^{2}$ Department of Laboratory Medicine \& Pathobiology, University of Toronto, Toronto, ON, Canada M5G 0A4 \\ ${ }^{3}$ Pulmonary \& Critical Care Medicine Division, Brigham \& Women's Hospital, Harvard Medical School, Boston, MA 02115, USA \\ ${ }^{4}$ Division of Respiratory Medicine, Department of Paediatrics, The Hospital for Sick Children, Toronto, ON, Canada M5G 1X8 \\ ${ }^{5}$ Institute of Medical Sciences, University of Toronto, Toronto, ON, Canada M5S 1A8 \\ ${ }^{6}$ Department of Pharmacology and Toxicology, University of Toronto, Toronto, ON, Canada M5S 1A8
}

Correspondence should be addressed to Nades Palaniyar; nades.palaniyar@sickkids.ca

Received 6 November 2014; Accepted 12 January 2015

Academic Editor: Eduardo López-Collazo

Copyright (C) 2015 David N. Douda et al. This is an open access article distributed under the Creative Commons Attribution License, which permits unrestricted use, distribution, and reproduction in any medium, provided the original work is properly cited.

\begin{abstract}
Pulmonary exacerbations in cystic fibrosis airways are accompanied by inflammation, neutrophilia, and mucous thickening. Cystic fibrosis sputum contains a large amount of uncleared DNA contributed by neutrophil extracellular trap (NET) formation from neutrophils. The exact mechanisms of the induction of NETosis in cystic fibrosis airways remain unclear, especially in uninfected lungs of patients with early cystic fibrosis lung disease. Here we show that Hepoxilin A3, a proinflammatory eicosanoid, and the synthetic analog of Hepoxilin B3, PBT-3, directly induce NETosis in human neutrophils. Furthermore, we show that Hepoxilin A3-mediated NETosis is NADPH-oxidase-dependent at lower doses of Hepoxilin A3, while it is NADPH-oxidase-independent at higher doses. Together, these results demonstrate that Hepoxilin A3 is a previously unrecognized inducer of NETosis in cystic fibrosis lungs and may represent a new therapeutic target for treating cystic fibrosis and other inflammatory lung diseases.
\end{abstract}

\section{Introduction}

In infected and inflamed tissues, recruited neutrophils release neutrophil extracellular traps (NETs) [1]. NETs are made of DNA, and the elaborate strings of DNA are decorated with histones and antimicrobial proteins and peptides [2]. While it was originally thought that NETs are an effective method by which neutrophils trap and kill bacteria [3], a number of studies now show the deleterious "side-effects" of NETs, especially when overproduced [4].

It is now known that NETs directly cause host cell death [5] and are directly linked to the pathogenesis of a number of lung disorders including transfusion related acute lung injury (TRALI) [6, 7], ventilator induced lung injury (VILI) [8], pneumonia $[9,10]$, and cystic fibrosis (CF) [11]. However, the precise mechanisms leading to the excess neutrophil recruitment, activation, and NET production are not clearly understood. Thus, the negative impact of NETs demonstrated in a variety of inflammatory disorders illustrates the need to better understand NETosis and its signaling pathways and physiological mechanisms in regulating NETosis in health.

Hepoxilin A3 (HxA3) is a hydroxyepoxide derivative of arachidonic acid [12] and is formed by a variety of tissues through the 12-lipoxygenase/hepoxilin synthase pathways [13]. A recent report shows that HxA3 is produced by epithelial cells in response to bacterial infection [14]. Furthermore, HxA3 is a chemoattractant that is necessary and sufficient to recruit neutrophils to infected and inflamed sites $[14,15]$. HxA3 causes the mobilization of intracellular calcium into the cytosol in neutrophils [16]. The increase in intracellular calcium causes activation of potassium current [17]. However, it is not known whether $\mathrm{HxA} 3$ can activate neutrophils to induce calcium dependent NETosis.

In this study, we sought to determine whether $\mathrm{HxA} 3$ can directly induce NETosis. We show that HxA3 and PBT-3, a synthetic analogue of $\mathrm{HxB} 3$, directly induce NETosis. These 


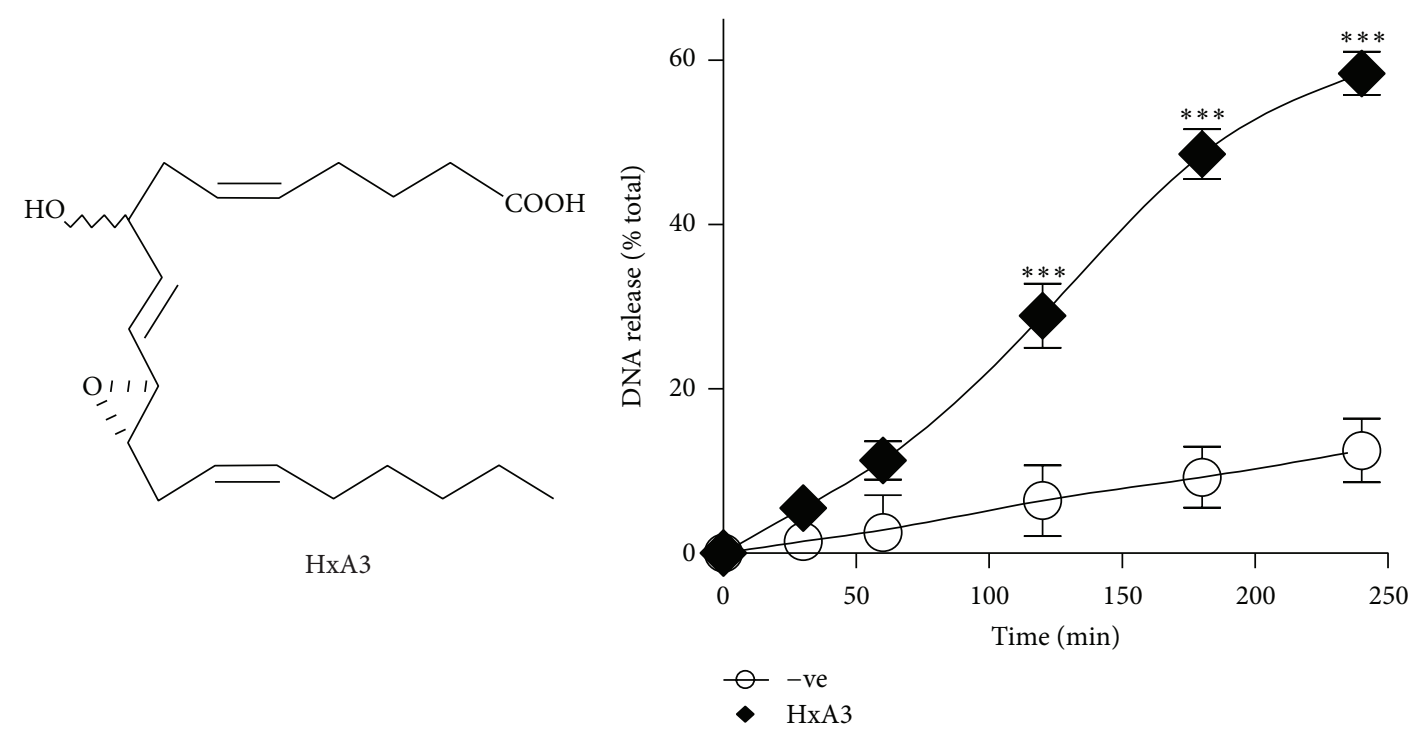

(a)

(b)

FIGURE 1: Hepoxilin A3 induces DNA release from human neutrophils. (a) Structure of HxA3. (b) Neutrophils were seeded into 96-well plates in the presence or absence of HxA3, and the extracellular DNA release was monitored using Sytox Green cell impermeable DNA dye. The results show a time-dependent increase in NET release after activation with $\mathrm{HxA} 3(10 \mu \mathrm{g} / \mathrm{mL})(n=4)$. HxA3 was used as the methyl ester.

results were confirmed by plate reader assays as well as by immunofluorescence assays. Using DPI $(20 \mu \mathrm{M})$, a specific inhibitor of NADPH oxidase (NOX), we also demonstrate that HxA3-induced NETosis is NOX-dependent at lower concentrations of $\mathrm{HxA} 3$ but becomes NOX-independent at higher doses of HxA3. Therefore, HxA3 is a novel member of natural lipid NET-inducers.

\section{Materials and Methods}

2.1. Reagents. All reagents were purchased from Sigma unless otherwise stated.

2.2. Hepoxilin A3 and PBT-3. Both $\mathrm{HxA} 3$ and the $\mathrm{Hx}$ analogue, PBT-3, were produced through total chemical synthesis and used in this study as the methyl ester. HxA3 was provided by Corey and Su [18] and PBT-3 was synthesized in our laboratory by Demin and Pace-Asciak [19]. Stock solutions of the compounds were stored in benzene at $-80^{\circ} \mathrm{C}$. When required, aliquots were taken, the solvent was removed with a stream of $\mathrm{N} 2$ gas, and the residue was dissolved in DMSO at a concentration of $1 \mu \mathrm{g} / \mu \mathrm{L}$ for $\mathrm{HxA} 3$ and $2 \mu \mathrm{g} / \mu \mathrm{L}$ for PBT-3 and added to the cells.

2.3. Human Peripheral Neutrophils. Peripheral blood from healthy donors was collected in K2 EDTA blood collection tubes (BD, Franklin Lakes, NJ). A signed informed consent was obtained from each donor, and the protocol was approved by the Hospital for Sick Children ethics committee. Neutrophils were then isolated from whole blood using PolymorphPrep (Axis-Shield PoC, Oslo, Norway) according to the manufacturer's instructions with minor modifications. The modifications are as follows: (i) the lysis of red blood cells was done using a hypotonic saline solution $(0.2 \%(\mathrm{w} / \mathrm{v}) \mathrm{NaCl})$ and was followed by the addition of an equal volume of $1.6 \%$ $(\mathrm{w} / \mathrm{v}) \mathrm{NaCl}$ solution with Hepes buffer $(20 \mathrm{mM}, \mathrm{pH} 7.2)$ to make the solution buffered and isotonic. This was followed by two washes with a wash buffer containing $0.85 \%$ (w/v) $\mathrm{NaCl}$ and Hepes (10 mM, pH 7.2). Isolated neutrophils were then resuspended in RPMI (Invitrogen) supplemented with Hepes buffer (10 mM, pH 7.2). In order to assess the requirement of NADPH oxidase activity, neutrophils were preincubated with NOX inhibitor diphenyleneiodonium (DPI) $(20 \mu \mathrm{M})$ for $1 \mathrm{~h}$ prior to the activation of cells for NETosis. For each experiment, only 1-2 donor samples were processed each day. The assays were repeated with different donors to obtain experimental replicates.

2.4. Plate Reader Assay. For the plate reader assay, cells were seeded at $3 \times 10^{4}$ cells per well in a 96-well plate in the culture media in the presence of Sytox Green cell impermeable nucleic acid stain (Life Technologies, Ontario, Canada) at $5 \mu \mathrm{M}$. The fluorescence was measured using POLARstar OMEGA fluorescence microplate reader (BMG Labtech, Ortenberg, Germany) at specific time intervals for up to $240 \mathrm{~min}$. To calculate the relative release of NET DNA, fluorescence readout obtained from cells lysed with $0.5 \%(\mathrm{v} / \mathrm{v})$ Triton X-100 was considered as $100 \%$ DNA release, and the release of NET DNA at each time point was expressed as the \% of total release.

2.5. Confocal Imaging. For imaging, cells used for the plate reader assay were fixed with PFA $(4 \%$, w/v) in PBS buffer for $10 \mathrm{~min}$ and permeablized with Triton X-100 $(0.1 \%$, w/v). Mouse- $\alpha$-myeloperoxidase antibody (ab25989, Abcam, Ontario, Canada) at 1:500 dilution and an anti-mouse IgG 

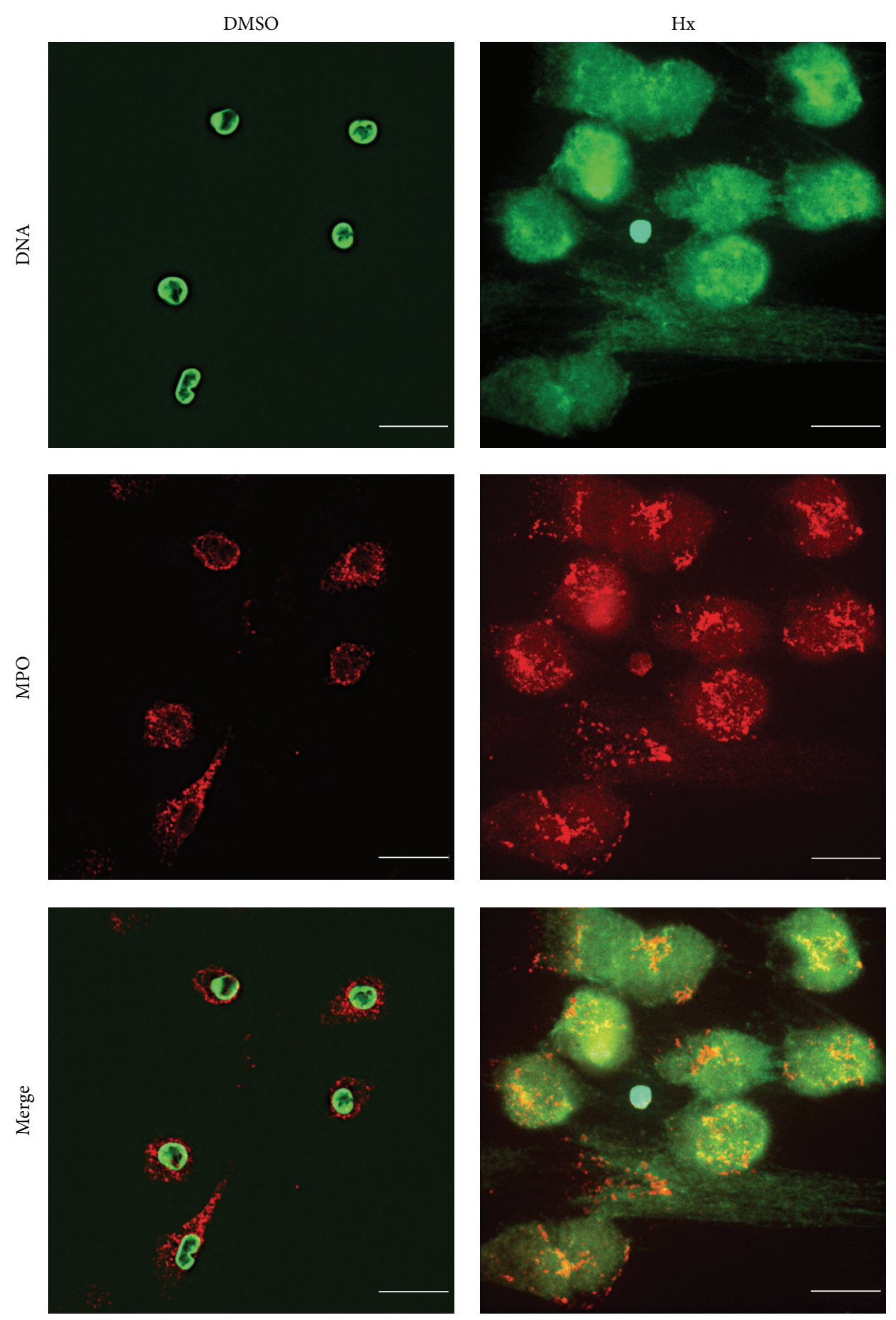

FIgURE 2: Hepoxilin A3 induces NETosis. Four hours after stimulation with either vehicle or HxA3, cells were fixed and stained for immunofluorescence analysis. Cells were stained for DNA (green) and MPO (red) after activation with $\mathrm{HxA} 3$ (5 $\mathrm{g} / \mathrm{mL}$ ). Images are representative of 3 independent experiments.

secondary antibody conjugated with Alexa Fluor 555 (Invitrogen) were used for staining MPO. DNA was already stained with Sytox Green for the plate reader assay. The confocal images were taken using the Olympus IX81 inverted fluorescence microscope equipped with a Hamamatsu C910013 back-thinned EM-CCD camera and Yokogawa CSU X1 spinning disk confocal scan head (with Spectral Aurora Borealis upgrade) and 4 separate diode-pumped solid state laser lines (Spectral Applied Research, $405 \mathrm{~nm}, 491 \mathrm{~nm}, 561 \mathrm{~nm}$, and $642 \mathrm{~nm}$ ). The objectives used were $20 \times / 0.75$ or $60 \times / 1.35$. The microscope was operated with Volocity software (Perkin Elmer, Waltham, MA). Images taken on the spinning disk 


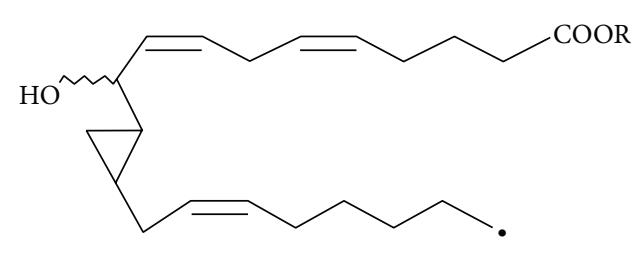

PBT-3

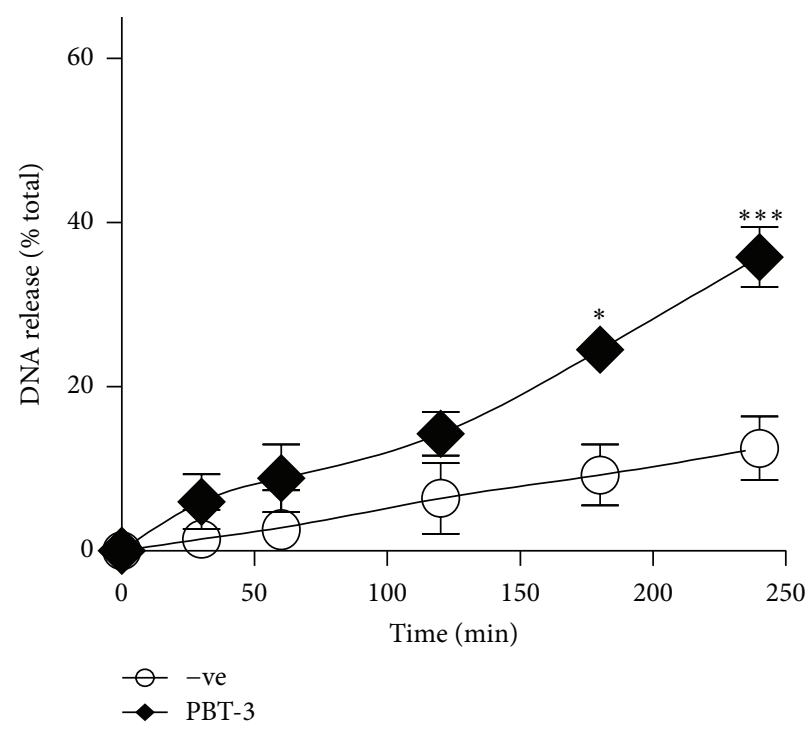

(b)

Figure 3: PBT-3 induces DNA release from human neutrophils. (a) Structure of PBT-3. (b) Neutrophils were seeded into 96-well plates in the presence or absence of PBT-3 $(20 \mu \mathrm{g} / \mathrm{mL})$, and the extracellular DNA release was monitored using Sytox Green cell impermeable DNA dye. The results show a time-dependent increase in NET release after activation with PBT-3 $(20 \mu \mathrm{g} / \mathrm{mL})(n=4)$. R = Methyl.

confocal microscope were deconvolved by iterative restoration with confidence limit set to $95 \%$ and iteration limit set to 20. All images were deconvolved to the confidence limit before reaching iteration limit.

2.6. Statistical Analysis. All data are presented as mean \pm sem. Statistical analysis was performed using GraphPad Prism statistical analysis software (Version 5.0a for Mac OS X). Where appropriate, ANOVA with Bonferroni post-hoc test was used. A $P$ value which was set at 0.05 or less was considered to be statistically significant.

\section{Results and Discussion}

3.1. Hepoxilin A3 Induces NETosis. A number of reports now suggest pathogenic roles for NETs in lung disorders [6, 7]. NETs have been shown to be associated with transfusionrelated acute lung injury (TRALI) in an experimental mouse model, as well as in humans by two independent groups $[6,7]$. In both of these studies, NETs were found to be present in circulation and in lungs $[6,7]$, and therapeutic strategies using DNase $[6,7]$ or anti-histone antibody [6] to target NETs were found to be protective. The damaging effect of NETs is thought to come directly from NETs, as it has been found that NETs are capable of inflicting injury to epithelial and endothelial cells $[5,20,21]$.

Here we sought to determine whether HxA3 directly induces NETosis in human neutrophils. HxA3 is an eicosanoid that acts as a lipid mediator of proinflammatory response $[12,15,16]$. In addition, our group has shown that HxA3 activates neutrophils and induces the release of intracellular calcium $[16,22]$. Because calcium signaling is a critical component of NETosis, we asked whether HxA3 is a natural inducer of NETosis. The plate reader assays demonstrate a timedependent NET release in response to HxA3 $(10 \mu \mathrm{g} / \mathrm{mL} ; P<$ 0.001 ; Figure 1). Immunofluorescence imaging confirms that HxA3 induces NET formation and release and that HxA3induced NETs contain myeloperoxidase (MPO) (Figure 2).

We next sought to confirm this finding by using PBT-3, a synthetic analogue of $\mathrm{HxB} 3[13,19]$. As expected, the plate reader assays demonstrate that NETs are released in response to PBT-3 (Figure 3). The plate reader assays also show that NET release by PBT-3 $(20 \mu \mathrm{g} / \mathrm{mL})$ increases over time $(P<$ $0.001)$; however, the extent of NET release was not as high as that induced by $\mathrm{HxA} 3$ at $240 \mathrm{~min}$ (Figure 1). This is not unexpected as PBT-3 is an analog of $\mathrm{HxB} 3$ (not HxA3), which is weak in releasing intracellular calcium from neutrophils. This strongly suggests that calcium plays an important role in mediating NETosis induced by HxA3. Immunofluorescence assays were then performed and confirmed that PBT- 3 indeed induces NETosis as evidenced by the colocalization of extracellular DNA and MPO (Figure 4).

3.2. HxA3-Mediated NETosis Is Both NOX-Dependent and NOX-Independent Depending on HxA3 Concentration. We next asked whether HxA3-induced NETosis was NOXdependent. The plate reader assays show that, at lower concentrations of $\mathrm{HxA} 3(2.5$ and $5 \mu \mathrm{g} / \mathrm{mL})$, DPI significantly reduces NET release, suggesting that, at these concentrations, ROS generation by NOX is required (Figure 5). At higher concentrations of $\mathrm{HxA} 3$, there is less suppression of NETosis by DPI $(P<0.05$; Figure 5$)$. At the highest concentration tested $(10 \mu \mathrm{g} / \mathrm{mL})$, HxA3-mediated NETosis completely overcomes the suppression by DPI and becomes NOX-independent $(P<0.001$, Figure 5$)$. These results suggest that 

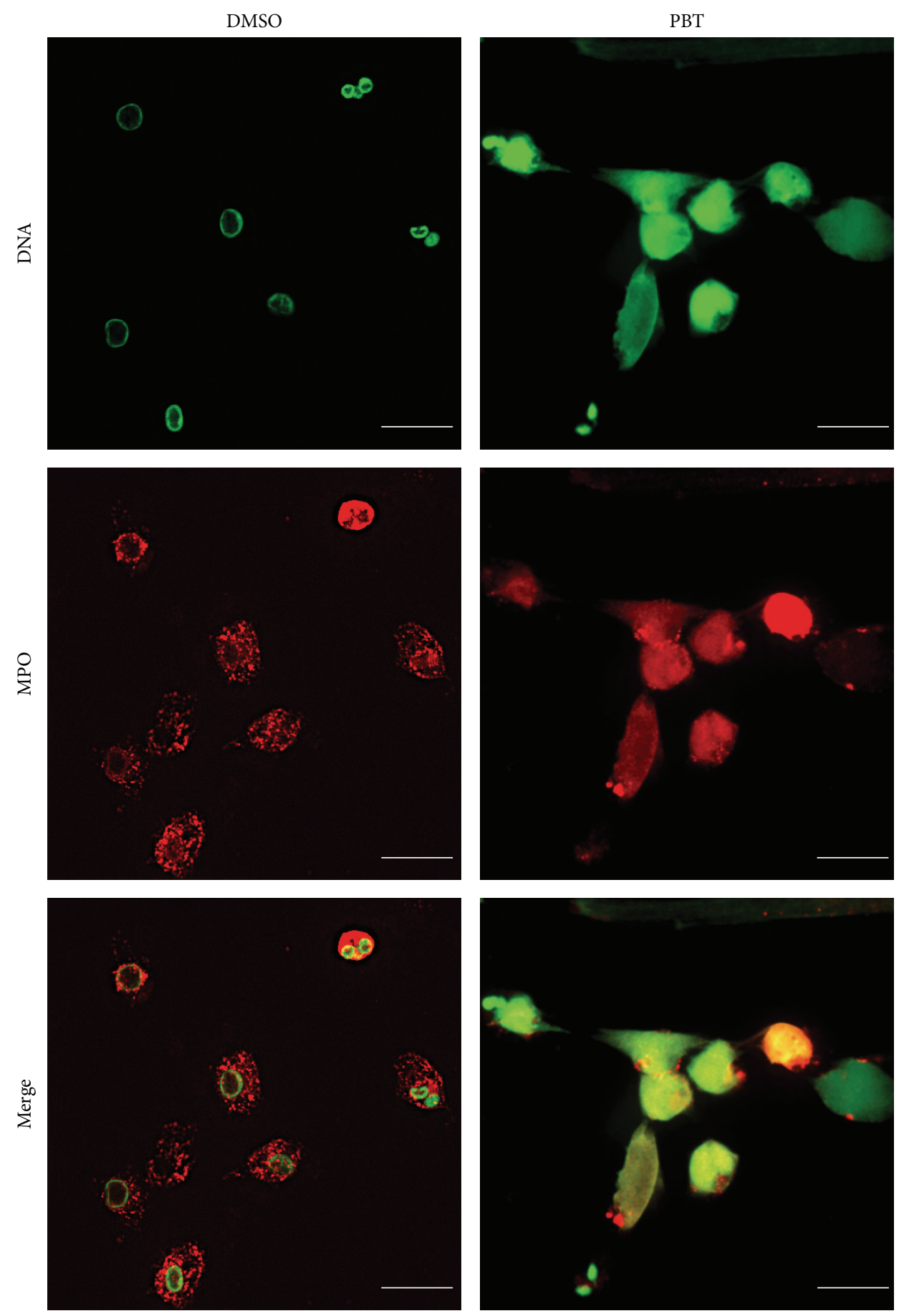

FIGURE 4: PBT-3 induces NETosis. Four hours after stimulation with either vehicle or PBT-3 (20 $\mu \mathrm{g} / \mathrm{mL})$, cells were fixed and stained for immunofluorescence analysis. Cells were stained for DNA (green) and MPO (red) after activation with PBT-3 (20 $\mu \mathrm{g} / \mathrm{mL}$ ). Images are representative of 3 independent experiments.

HxA3-mediated NETosis has both NOX-dependent and NOX-independent features, conditioned by the HxA3 concentration.

Our group has shown previously that $\mathrm{HxA} 3$ activates neutrophils by inducing a rise in intracellular calcium concentration [16]. Furthermore, we have shown that the activation of neutrophils with $\mathrm{HxA} 3$ promotes the release of diacylglycerol
(DAG) in addition to increasing intracellular calcium [23], pointing to the possibility that HxA3 activates PKC as well as calcium signaling. Of the two distinct forms of NETosis (i.e., NOX-dependent and NOX-independent pathways), calcium is indispensable in the NOX-independent pathway [24]. Furthermore, the activation of NOX-independent pathway in neutrophils results in a rapid release of NETs $(<1 \mathrm{~h})$ compared 


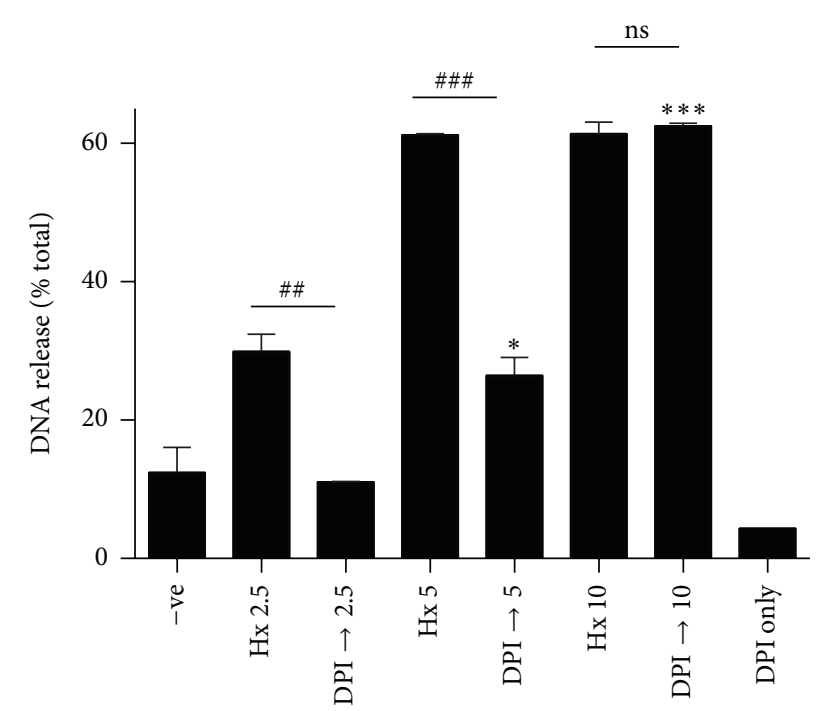

FIgUre 5: Hepoxilin A3-mediated NETosis is both NOX-dependent and NOX-independent depending on the dose of HxA3. A fluorescence plate reader assay was utilized to monitor NET DNA release upon stimulation with $\mathrm{HxA} 3$ in the presence or absence of the NOX inhibitor DPI $(20 \mu \mathrm{M})\left(n=2 ;{ }^{*} P<0.05 ;{ }^{* * *} P<0.001\right.$ compared to cells activated with $\mathrm{HxA} 3(2.5 \mu \mathrm{g} / \mathrm{mL})$ in the presence of DPI $\left(\right.$ DPI $\rightarrow 2.5$ ); ${ }^{\# \# ~} P<0.01 ;{ }^{\# \# \# ~} P<0.001$ (one-way ANOVA)).

to the NOX dependent pathway $(>2 \mathrm{~h})$. Thus, it is possible that NOX-dependent and NOX-independent NETosis are conditioned by $\mathrm{HxA} 3$, whereby the activation of a specific pathway depends on $\mathrm{HxA} 3$ concentration.

As mentioned above, NETs have been shown to be involved in a number of inflammatory lung disorders [4]. Although some mechanistic insights have been obtained for lung disorders such as TRALI, VILI, and pneumonia [6-10], the precise mechanisms leading to abundant NET production in other conditions such as CF remain elusive. It has been shown that NETs are found in airways of patients with CF [11]. Bacterial pathogens such as Pseudomonas aeruginosa commonly infect CF airways. The overabundance of NET DNA can support the biofilm formation by $P$. aeruginosa [25-27]. Because hepoxilins can be produced by epithelial cells in response to $P$. aeruginosa infection [14], act as a chemoattractant for neutrophils $[14,15,28]$, and directly induce NETosis (Figures 1 and 2), these compounds can be important therapeutic targets for NET-associated lung disorders.

\section{Conclusions}

The present study demonstrates that HxA3 can induce NETosis in human neutrophils. The results were corroborated by using PBT-3, a synthetic analogue of $\mathrm{HxB} 3$. The requirement for ROS generation by NOX in HxA3-mediated NETosis is dose-dependent, suggesting that HxA3 utilizes both NOXdependent and NOX-independent pathways. Our finding that $\mathrm{HxA} 3$ directly induces NETosis suggests that $\mathrm{HxA} 3$ could induce NETosis in the lung, and hepoxilins and members of hepoxilin-generating pathways may therefore be therapeutic targets in NETs related inflammatory lung disorders.

\section{Conflict of Interests}

None of the authors has any conflict of interests relevant to this paper.

\section{Authors' Contribution}

David N. Douda designed and carried out experiments, analyzed data, and wrote the paper. Hartmut Grasemann participated in experimental design and paper editing. Cecil PaceAsciak provided HxA3 and PBT-3 and was involved in the project concept, discussions, and paper editing. Nades Palaniyar is the principal investigator who conceived the project and was involved in experimental design and data analysis and paper editing.

\section{Acknowledgments}

David N. Douda was supported by Ontario Graduate Scholarship, Ontario Student Opportunity Trust Fund (SickKids Restracomp, Dr. Goran Enhorning Award in Pulmonary Research, and Peterborough K.M. Hunter Graduate Studentship), and University of Toronto Doctoral Thesis Completion Grant. The study was funded by CIHR (MOP-134761) and CCFF (2619) Grants to Nades Palaniyar.

\section{References}

[1] A. Mócsai, "Diverse novel functions of neutrophils in immunity, inflammation, and beyond," The Journal of Experimental Medicine, vol. 210, no. 7, pp. 1283-1299, 2013.

[2] V. Brinkmann and A. Zychlinsky, "Neutrophil extracellular traps: is immunity the second function of chromatin?" The Journal of Cell Biology, vol. 198, no. 5, pp. 773-783, 2012.

[3] V. Brinkmann, U. Reichard, C. Goosmann et al., "Neutrophil extracellular traps kill bacteria," Science, vol. 303, no. 5663, pp. $1532-1535,2004$.

[4] O. Z. Cheng and N. Palaniyar, "NET balancing: a problem in inflammatory lung diseases," Frontiers in Immunology, vol. 4, article 1, 2013.

[5] M. Saffarzadeh, C. Juenemann, M. A. Queisser et al., "Neutrophil extracellular traps directly induce epithelial and endothelial cell death: a predominant role of histones," PLOS ONE, vol. 7, no. 2, Article ID e32366, 2012.

[6] A. Caudrillier, K. Kessenbrock, B. M. Gilliss et al., "Platelets induce neutrophil extracellular traps in transfusion-related acute lung injury," The Journal of Clinical Investigation, vol. 122, no. 7, pp. 2661-2671, 2012.

[7] G. M. Thomas, C. Carbo, B. R. Curtis et al., "Extracellular DNA traps are associated with the pathogenesis of TRALI in humans and mice," Blood, vol. 119, no. 26, pp. 6335-6343, 2012.

[8] J. Rossaint, J. M. Herter, H. van Aken et al., "Synchronized integrin engagement and chemokine activation is crucial in neutrophil extracellular trap-mediated sterile inflammation," Blood, vol. 123, no. 16, pp. 2573-2584, 2014.

[9] N. Branzk, A. Lubojemska, S. E. Hardison et al., "Neutrophils sense microbe size and selectively release neutrophil extracellular traps in response to large pathogens," Nature Immunology, vol. 15, no. 11, pp. 1017-1025, 2014. 
[10] T. Narasaraju, E. Yang, R. P. Samy et al., "Excessive neutrophils and neutrophil extracellular traps contribute to acute lung injury of influenza pneumonitis," The American Journal of Pathology, vol. 179, no. 1, pp. 199-210, 2011.

[11] M. Dwyer, Q. Shan, S. D’Ortona et al., "Cystic fibrosis sputum DNA has netosis characteristics and neutrophil extracellular trap release is regulated by macrophage migration-inhibitory factor," Journal of Innate Immunity, vol. 6, no. 6, pp. 765-779, 2014.

[12] C. R. Pace-Asciak and J. M. Martin, "Hepoxilin, a new family of insulin secretagogues formed by intact rat pancreatic islets," Prostaglandins, Leukotrienes and Medicine, vol. 16, no. 2, pp. 173-180, 1984.

[13] C. R. Pace-Asciak, "The hepoxilins and some analogues: a review of their biology," British Journal of Pharmacology, vol. 158, no. 4, pp. 972-981, 2009.

[14] B. P. Hurley, D. Siccardi, R. J. Mrsny, and B. A. McCormick, "Polymorphonuclear cell transmigration induced by Pseudomonas aeruginosa requires the eicosanoid hepoxilin $\mathrm{A}_{3}$," The Journal of Immunology, vol. 173, no. 9, pp. 5712-5720, 2004.

[15] B. A. McCormick, "Bacterial-induced hepoxilin $\mathrm{A}_{3}$ secretion as a pro-inflammatory mediator," The FEBS Journal, vol. 274, no. 14, pp. 3513-3518, 2007.

[16] S. Dho, S. Grinstein, E. J. Corey, W.-G. Su, and C. R. Pace-Asciak, "Hepoxilin A3 induces changes in cytosolic calcium, intracellular $\mathrm{pH}$ and membrane potential in human neutrophils," Biochemical Journal, vol. 266, no. 1, pp. 63-68, 1990.

[17] H. Berkefeld, B. Fakler, and U. Schulte, " $\mathrm{Ca}^{2+}$-activated $\mathrm{K}^{+}$ channels: from protein complexes to function," Physiological Reviews, vol. 90, no. 4, pp. 1437-1459, 2010.

[18] E. J. Corey and W. G. Su, "Total synthesis of biologically active metabolites of arachidonic acid. The two 8-hydroxy-11,12(S,S)epoxyeicosa-5,14(Z), 9(E)-trienoic acids," Tetrahedron Letters, vol. 25, no. 45, pp. 5119-5122, 1984.

[19] P. M. Demin and C. R. Pace-Asciak, "Synthesis of racemic 11,12-cyclopropyl analogs of hepoxilins A3 and B3," Tetrahedron Letters, vol. 34, no. 27, pp. 4305-4308, 1993.

[20] F. H. Pilsczek, D. Salina, K. K. H. Poon et al., "A novel mechanism of rapid nuclear neutrophil extracellular trap formation in response to Staphylococcus aureus," The Journal of Immunology, vol. 185, no. 12, pp. 7413-7425, 2010.

[21] E. Villanueva, S. Yalavarthi, C. C. Berthier et al., "Netting neutrophils induce endothelial damage, infiltrate tissues, and expose immunostimulatory molecules in systemic lupus erythematosus," Journal of Immunology, vol. 187, no. 1, pp. 538-552, 2011.

[22] D. Reynaud, P. M. Demin, M. Sutherland, S. Nigam, and C. R. Pace-Asciak, "Hepoxilin signaling in intact human neutrophils: biphasic elevation of intracellular calcium by unesterified hepoxilin A3," FEBS Letters, vol. 446, no. 2-3, pp. 236-238, 1999.

[23] S. Nigam, S. Nodes, G. Cichon, E. J. Corey, and C. R. PaceAsciak, "Receptor-mediated action of hepoxilin $\mathrm{A}_{3}$ releases diacylglycerol and arachidonic acid from human neutrophils," Biochemical and Biophysical Research Communications, vol. 171, no. 3, pp. 944-948, 1990.

[24] H. Parker, M. Dragunow, M. B. Hampton, A. J. Kettle, and C. C. Winterbourn, "Requirements for NADPH oxidase and myeloperoxidase in neutrophil extracellular trap formation differ depending on the stimulus," Journal of Leukocyte Biology, vol. 92, no. 4, pp. 841-849, 2012.

[25] Q. M. Parks, R. L. Young, K. R. Poch, K. C. Malcolm, M. L. Vasil, and J. A. Nick, "Neutrophil enhancement of Pseudomonas aeruginosa biofilm development: human F-actin and DNA as targets for therapy," Journal of Medical Microbiology, vol. 58, part 4, pp. 492-502, 2009.

[26] T. S. Walker, K. L. Tomlin, G. S. Worthen et al., "Enhanced Pseudomonas aeruginosa biofilm development mediated by human neutrophils," Infection and Immunity, vol. 73, no. 6, pp. 36933701, 2005.

[27] R. B. Thornton, S. P. Wiertsema, L.-A. S. Kirkham et al., "Neutrophil extracellular traps and bacterial biofilms in middle ear effusion of children with recurrent acute otitis media-a potential treatment target," PLoS ONE, vol. 8, no. 2, Article ID e53837, 2013.

[28] D. L. Tamang, W. Pirzai, G. P. Priebe et al., "Hepoxilin $A_{3}$ facilitates neutrophilic breach of lipoxygenase-expressing airway epithelial barriers," Journal of Immunology, vol. 189, no. 10, pp. 4960-4969, 2012. 


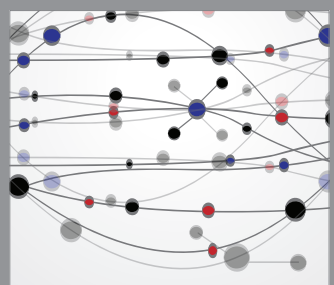

The Scientific World Journal
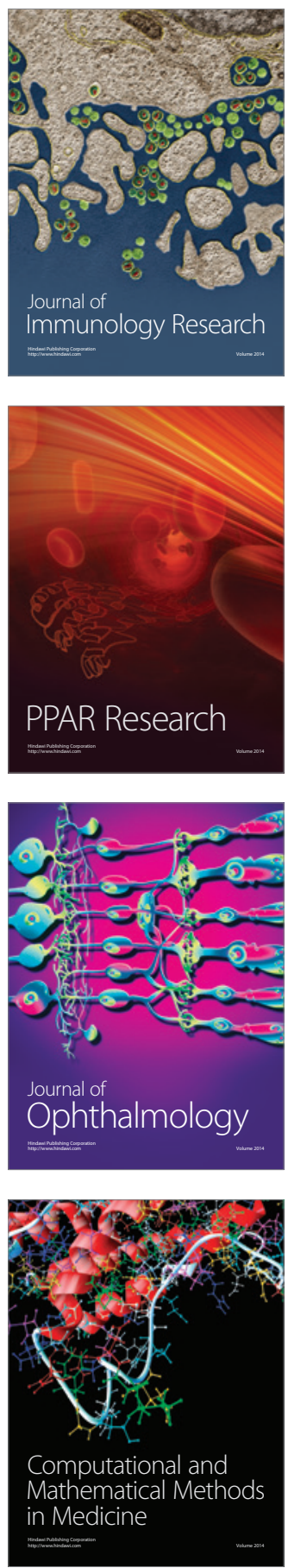

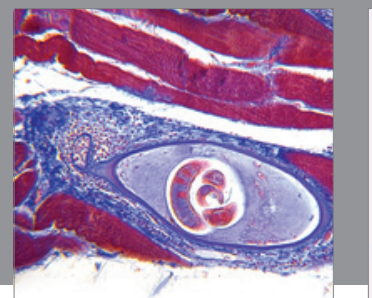

Gastroenterology

Research and Practice
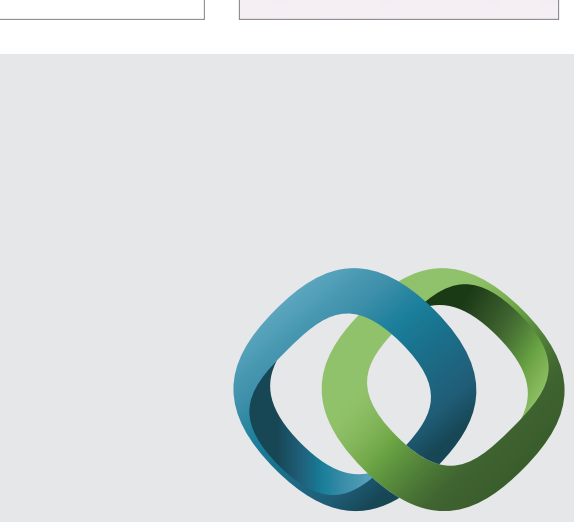

\section{Hindawi}

Submit your manuscripts at

http://www.hindawi.com
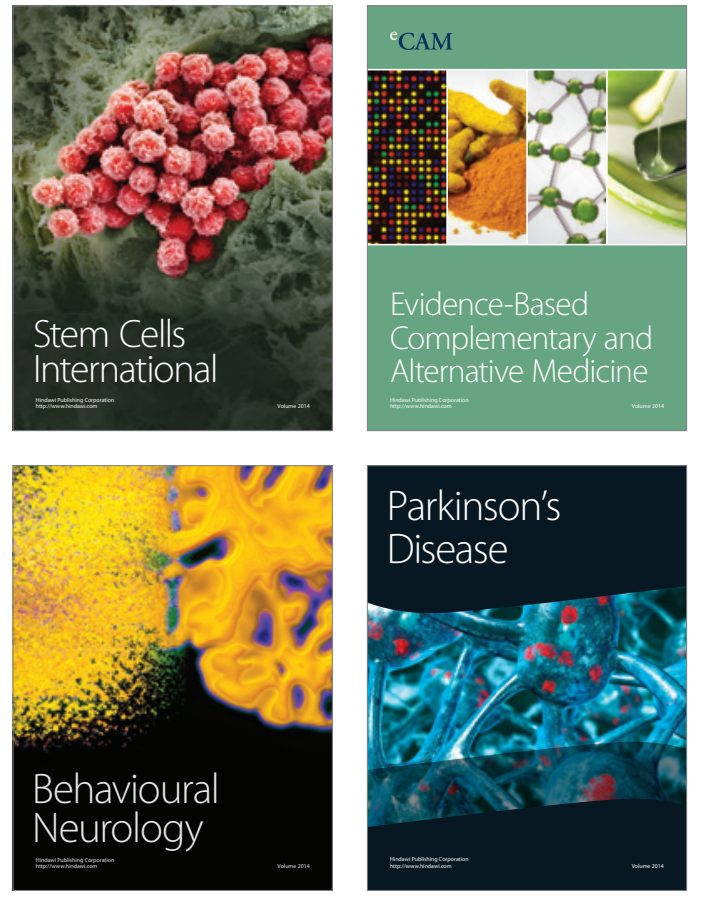
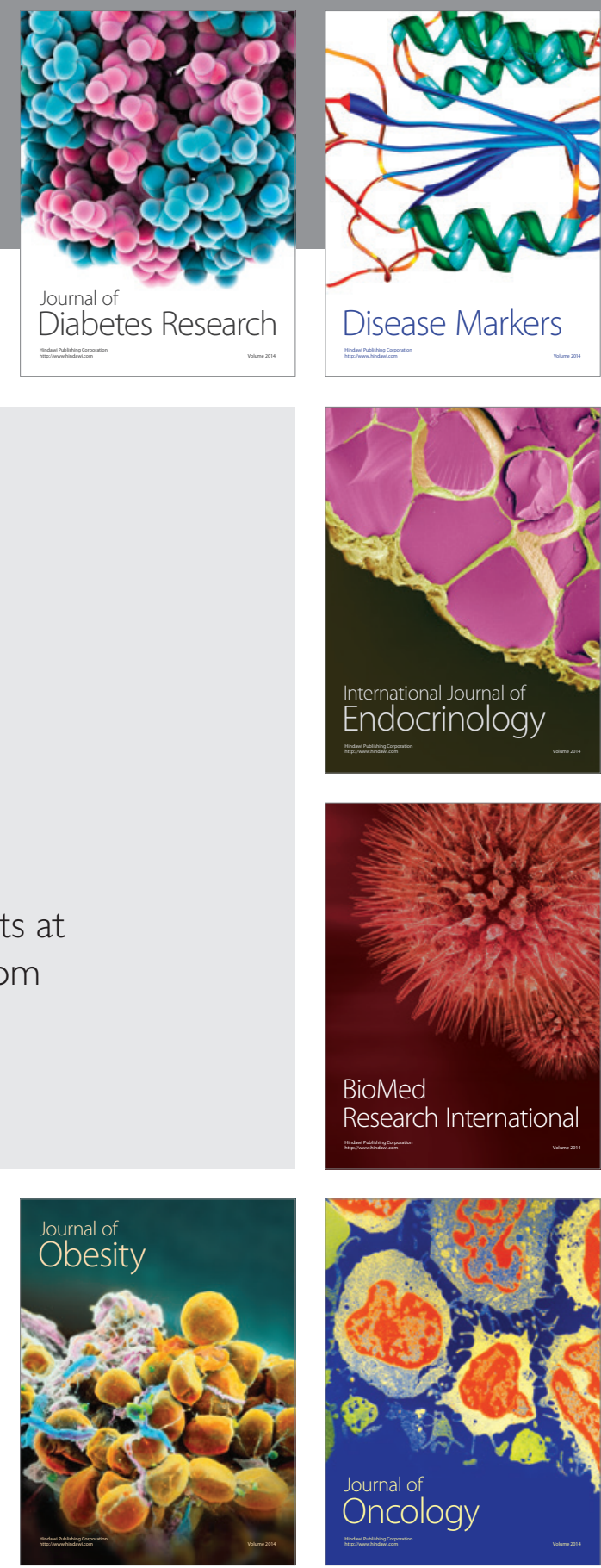

Disease Markers
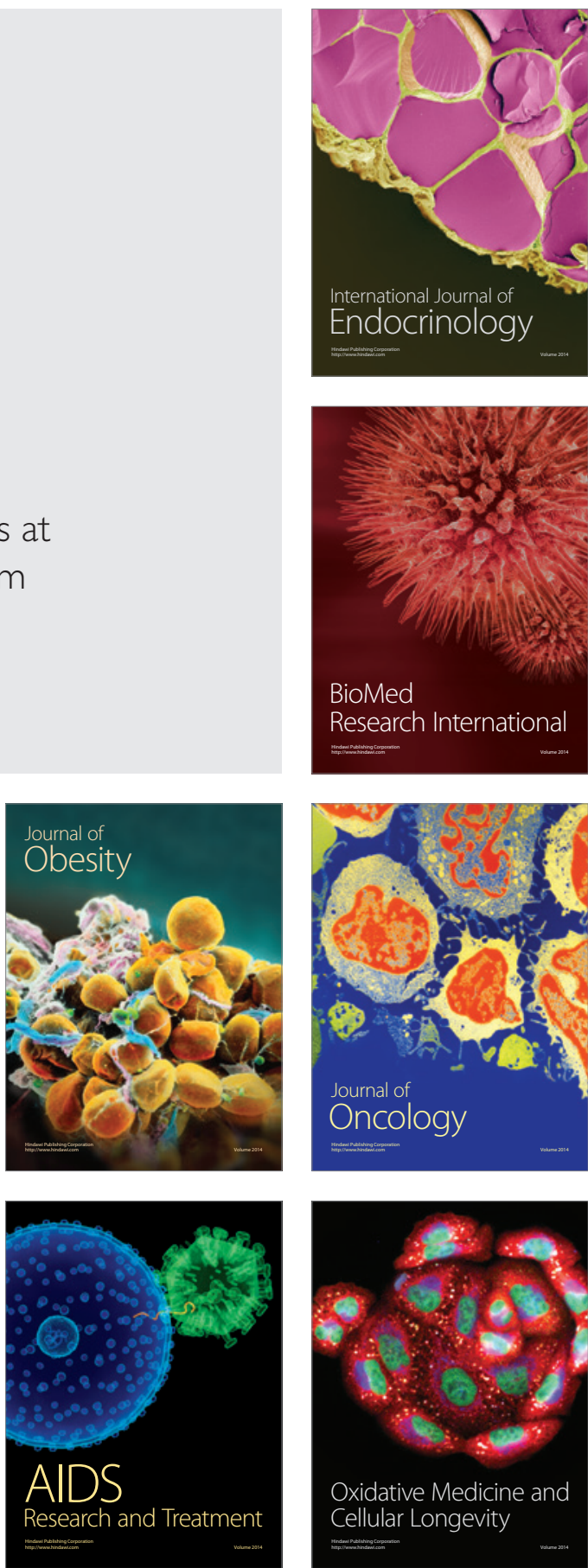